\title{
Experience Gained From the Construction, Test and Operation of the Large 4-T CMS Coil
}

\author{
Alain Hervé, Domenico Campi, Benoît Curé, Pasquale Fabbricatore, Andrea Gaddi, François Kircher, and \\ Stefano Sgobba
}

\begin{abstract}
The 4-T, 6-m free bore CMS solenoid has been successfully tested, operated and mapped at CERN during the autumn of 2006; R\&D studies started in 1993 and the construction proper in 1997. The main parameters of this 100 MUS\$ project (including yoke) were then considered beyond what was thought possible, as the total stored magnetic energy reaches $2.6 \mathrm{GJ}$ for a specific magnetic energy density exceeding $11 \mathrm{~kJ} / \mathrm{kg}$ of cold mass. During this period, the international design and construction team had to make several important technical choices, in particular mechanical, to maximize the chances of reaching the nominal induction of 4 Tesla. The paper will review these choices in the light of what is presently known and examine if better solutions would be possible today for constructing a new large high-field thin solenoid for a future detector magnet.
\end{abstract}

Index Terms-CMS, detector magnets, solenoids, superconducting magnets.

\section{INTRODUCTION}

T HE CMS experiment (Compact Muon Solenoid) is a general-purpose proton-proton detector designed to run at the highest luminosity at the CERN Large Hadron Collider (LHC). Its distinctive features include a high-magnetic-field solenoid (4 T) coupled with a multilayer muon system, a fully active scintillating-crystal electromagnetic calorimeter, a tile hadronic calorimeter, and a powerful inner tracking system. The most important aspect of the overall detector design is the configuration and parameters of the magnetic field for the measurement of muon momenta. The requirement for good momentum resolution, without making stringent demands on the spatial resolution of muon chambers, leads naturally to the choice of a high solenoidal magnetic field. A long superconducting solenoid $(12.5 \mathrm{~m})$ has been chosen with a free inner diameter of $6 \mathrm{~m}$ and a uniform magnetic induction of $4 \mathrm{~T}$. The muon spectrometer then consists of a single magnet allowing for a simpler architecture of the detector. The inner coil radius is large enough to accommodate the inner tracker and the full calorimetry. The magnetic flux is returned via a $1.5-\mathrm{m}$ thick saturated iron yoke instrumented with four stations of muon chambers.

The CMS experiment, which is being installed at a depth of $100 \mathrm{~m}$ below ground on the Interaction Point -5 of the LHC, is

Manuscript received August 22, 2007.

A. Hervé, D. Campi, B. Curé, A. Gaddi, and S. Sgobba are with CERN, Geneva, Switzerland (e-mail: alain.herve@cern.ch).

P. Fabbricatore is with INFN-Genova, Italy.

F. Kircher is with CEA/DAPNIA, Saclay, France.

Color versions of one or more of the figures in this paper are available online at http://ieeexplore.ieee.org.

Digital Object Identifier 10.1109/TASC.2008.920526

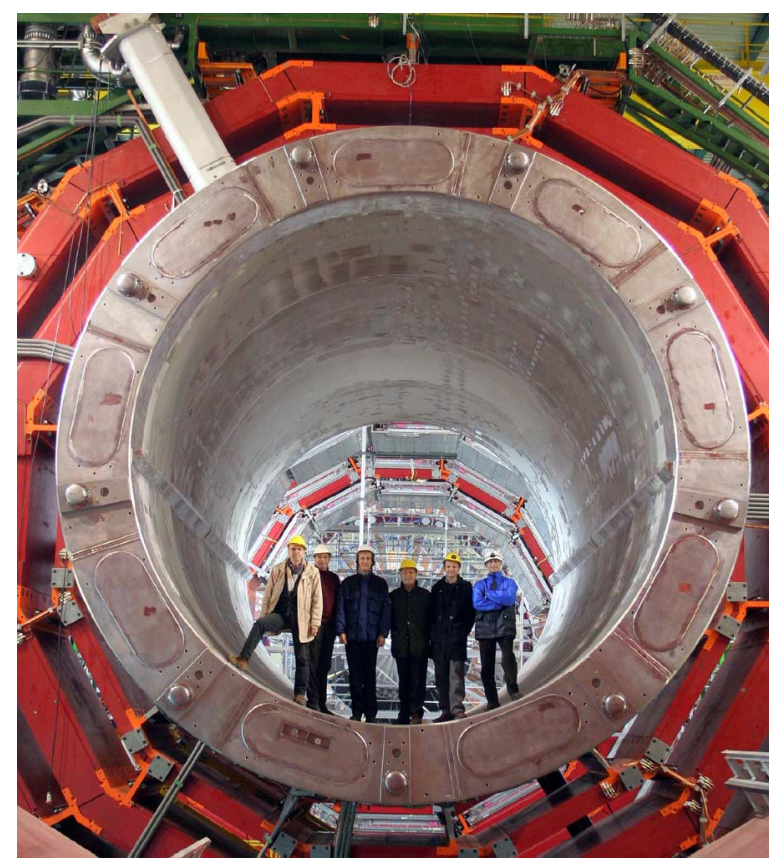

Fig. 1. A view of the 6-m free bore 4-T CMS solenoid, in the surface hall at Point- 5 of the LHC, before the magnetic test in the autumn of 2006.

built and funded by an international collaboration of High Energy Physics institutes from 38 countries $^{1}$ and by CERN (Fig. 1).

The CMS magnet [1] is the backbone of the CMS experiment as all sub-detectors are supported from it. The 100 MUS\$ magnet is a "common project" of the collaboration but several institutes have taken a direct technical participation in the construction of the magnet according to their scientific and technological interests, in particular CEA Saclay, ETH Zürich, Fermilab, INFN Genova, ITEP Moscow, University of Wisconsin and CERN.

The magnet has been assembled [2], tested [3], [4] and mapped [5] in a surface hall during the autumn 2006, and it is being lowered in the underground area by heavy lifting means. This bold choice has decoupled the work on the magnet assembly and test from the construction of the experimental area. The magnet will be re-commissioned underground during Spring 2008.

\footnotetext{
${ }^{1}$ Armenia, Austria, Belarus, Belgium, Brazil, Bulgaria, China, Columbia, Croatia, Cyprus, Estonia, Finland, France, Georgia, Germany, Greece, Hungary, India, Iran, Ireland, Italy, Korea, Lithuania, Mexico, New-Zealand, Pakistan, Poland, Portugal, Russia, Serbia, Spain, Switzerland, Taiwan, Turkey, Ukraine, United Kingdom, United States of America and Uzbekistan
} 
TABLE I

MAIN PARAMETERS OF CMS COIL

\begin{tabular}{ll}
\hline \hline Parameter & Value \\
\hline Magnetic length & $12.5 \mathrm{~m}$ \\
Free bore diameter & $6.0 \mathrm{~m}$ \\
Central magnetic induction & $4.0 \mathrm{~T}$ \\
Maximum induction on conductor & $4.6 \mathrm{~T}$ \\
Nominal current & $19.1 \mathrm{kA}$ \\
Average inductance & $14.2 \mathrm{H}$ \\
Stored energy & $2.6 \mathrm{GJ}$ \\
Stored energy per unit of cold mass & $11.6 \mathrm{~kJ} / \mathrm{kg}$ \\
Operating temperature & $4.5 \mathrm{~K}$ \\
\hline \hline
\end{tabular}

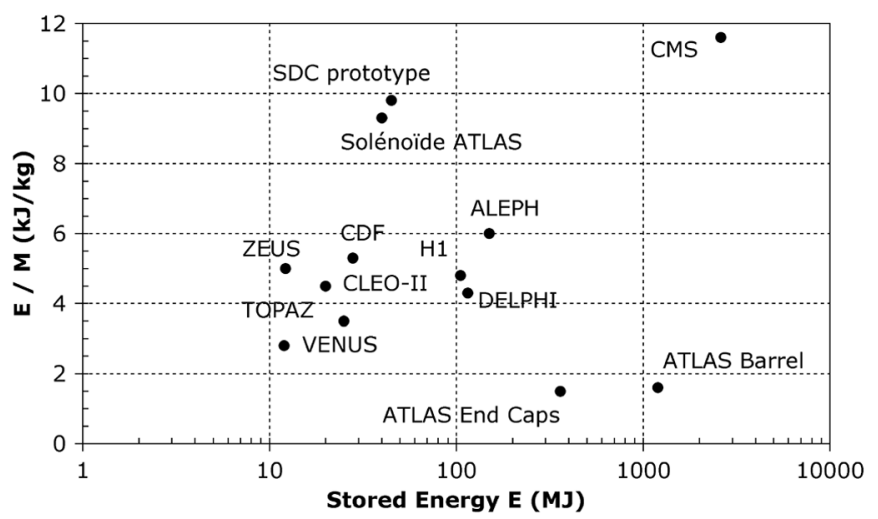

Fig. 2. Density of stored magnetic energy per unit of cold mass for several detector magnets.

The coil is an indirectly-cooled, aluminum-stabilized, fourlayer superconducting thin solenoid built in five modules. Its main parameters are given in Table I.

For physics reasons, the radial extent of the coil had to be kept small, and thus the CMS coil is in effect a "thin coil". The thickness of the coil is $3.9 \mathrm{X}_{0}$ (radiation length) and the specific energy ratio is $11.6 \mathrm{~kJ} / \mathrm{kg}$ of cold mass. As seen in Fig. 2, this parameter compares favorably with other thin coils.

The coil has been wound using the inner winding method, and the external mandrels are used as quench-back cylinders providing intrinsic protection. The coil is indirectly cooled by saturated helium at $4.5 \mathrm{~K}$ circulating in the thermosiphon mode through a network of pipes welded to the external mandrels.

At the inception of the project, in 1990, the ALEPH solenoid [6] has been taken as a demonstrator, and good points were directly adopted:

1) Use of NbTi with pure-aluminum stabilization, and aluminum-alloy construction to prevent problems with differential thermal contraction.

2) Target $2 \mathrm{~K}$ for the stability margin.

3) Use an external mandrel to support an indirect cooling loop, and inner winding of the coil insulated with fiberglass epoxy resin, potted using vacuum impregnation.

4) Take advantage of the external mandrel to benefit of a passive protection scheme based on the quench-back effect.

5) Keep the mean temperature of the coil below $130 \mathrm{~K}$ if all the magnetic energy is taken by the enthalpy of the cold mass, in case of malfunctioning of the active elements of the protection system.
Nevertheless, numerous improvements (listed in the next section) were needed to match the challenge of increasing the central induction to $4 \mathrm{~T}$.

After the successful test and operation of the CMS coil, it is interesting to review the main technical choices made by the international design and construction team to maximize the chances of reaching the nominal induction of 4 Tesla.

As several similar, even more ambitious, coils are being proposed, it is important to examine if better solutions would be possible today for constructing a new large high-field thin solenoid for a future detector magnet, with central induction in the range of 4 to $5 \mathrm{~T}$.

\section{DeParting PARAMETERS}

The main departing parameters from the ALEPH design were connected to the central induction of $4 \mathrm{~T}$, namely:

- Even choosing a much higher current (20 kA), a four-layer construction in 5 modules is needed. This imposes new constraints on the quality of the winding process.

- The equivalent magnetic pressure of 64 bars acting on the coil necessitates the use of high-strength aluminum alloys for structural elements.

- The founding assumption of the CMS design introduced by J.C. Lottin [7] was to position this high-strength aluminum alloy in direct contact with the conductor to create, in effect, a compound reinforced conductor.

- The circumferential hoop-strain has to reach $0.15 \%$ to stay within a reasonable thickness for the cold mass $(310 \mathrm{~mm})$ so that the radial extent of the coil system including the vacuum tank is limited to $600 \mathrm{~mm}$.

- Combined with the hoop-stress, a very large axial magnetic force has to be transmitted from module to module.

- The stored magnetic energy being $2.6 \mathrm{GJ}$, the energy density per unit of cold mass reaches $11.6 \mathrm{~kJ} / \mathrm{kg}$, and this placed the coil away in the parameter space compared to previous superconducting solenoids (Fig. 2), and clearly innovative solutions were needed to meet this challenging positioning.

\section{Design Challenges at the IncePtion of the PRoJeCt}

The main design challenges have been identified, very early on, as the following:

- How to obtain a stabilized conductor having the necessary section to provide the $2 \mathrm{~K}$ stability margin? One must consider that the section of the stabilized superconducting element (the insert) is an order of magnitude larger than what had been produced up to 1990 .

- How to get a compound conductor having the necessary mechanical strength? Using a compound reinforced conductor has always been considered, by the design team, the only way to allow controlling strains, and keep low shear stress in the insulation. The fact that there is only one element to wind (with an excellent geometry due to the final in-line milling) eases the challenges of producing a high quality multi-layer winding. Also, if well chosen, the reinforcing alloy sections can even be hardened during the curing process; meaning that one obtains the ultimate elastic limit only after the winding process is completed. 


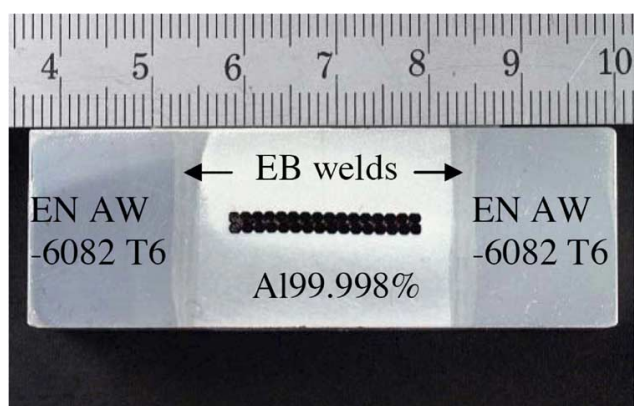

Fig. 3. Cross-section of the CMS conductor. The material grades used for the present conductor are shown in the picture. The EB weld seams join the insert and the reinforcement sections. The Rutherford cable is co-extruded with the insert. The insert (conductor) cross section is $30 \mathrm{~mm} \times 21.6 \mathrm{~mm}(64 \mathrm{~mm} \times 21.6$ $\mathrm{mm}$ ). The cross section of the NbTi-Cu stabilized Rutherford cable is $20.68 \mathrm{~mm}$ $\times 2.34 \mathrm{~mm}$. 21 lengths of $2.6 \mathrm{~km}$ of conductor have been produced for CMS.

- How to wind a very stiff conductor in four layers? The inertia of the compound conductor is very large compared to what had been done so far.

- How to build precise mandrels? Because of the high strain, it is not possible to thermally stress-relieve the mandrels to stabilize them; nevertheless, good cylindricity is required to obtain a precise coil.

- How to limit shear stresses inside the coil? The use of a reinforced conductor solves this problem inside the modules; however, in the region between two modules the large compression force must be transmitted without creating undue shear stresses.

- How to mount such a heavy coil (220 t) with a horizontal axis inside the vacuum tank?

\section{How the Design Challenges Have BeEn Met}

\section{A. Superconducting Insert}

Building on the pioneering work done for the first thin solenoids, the superconducting element of the conductor, the insert (Fig. 3), a NbTi Rutherford cable stabilized with pure aluminum in a continuous extrusion with the needed section, has been industrialized in the early 90s [8], and the process has been subsequently used not only for CMS but also for the conductor of the ATLAS toroids.

\section{B. Compound Conductor}

Identification of the best geometry for the compound reinforced conductor occupied most of the early effort and finally, as often, the simplest geometry proved to be the best one (Fig. 3).

How to attach the two reinforcement sections to the insert has been the next challenge. Ambitious solutions involving parallel co-extrusion were tried and abandoned. Continuous Electron-Beam (EB) welding [9], [10] was adopted because it satisfied fully the requirements, there was expertise in the team, and a competent medium size firm (TECHMETA), interested in the manufacturing challenge, was found nearby.

One difficulty has been to obtain the reinforcing sections in 2.6-km lengths with good mechanical uniformity, although they were not produced by continuous extrusion.

Several high-strength aluminum alloys in various tempers have been considered for the reinforcement. The typical alloy

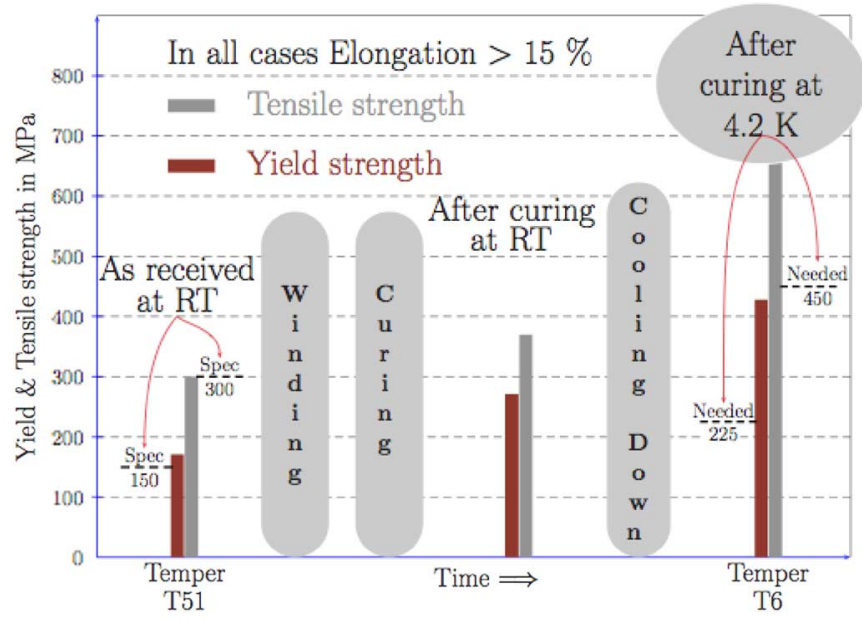

Fig. 4. Evolution of the mechanical properties of the alloy 6082 selected for the reinforcement of the conductor, from state T51 to T6 at RT and $4.2 \mathrm{~K}$.

EN AW-6082 has been selected for its high toughness, good extrudability and weldability. The underaged state (T51) allows winding to proceed with a not too stiff conductor, nevertheless resulting after the curing cycle to a T6 temper with further overall increase of the tensile yield strength, reaching at $4.2 \mathrm{~K}$ a $R p_{0.2}$ of $400 \mathrm{MPa}$ and a $R_{\mathrm{m}}$ of $650 \mathrm{MPa}$, largely sufficient for the application [11], [12] (Fig. 4).

\section{Selection of Material for the Mandrels}

To get aluminum plates with the necessary mechanical properties has been difficult. In fact, it seems impossible to build mandrels, with the required mechanical properties, thicker than $100 \mathrm{~mm}$ by welding plates together. This proved to be an a-posteriori justification for the use of the compound reinforced conductor.

An extensive and comparative characterization of the low temperature properties of different aluminum alloys and their welds resulted in the selection of the general-purpose EN AW-5083-H321 as the base material for the welded fabrication of the shells. The strain-hardened and stabilized temper H321 guarantees a safe yield-strength $\left(R p_{0.2}\right)$ of $254 \mathrm{MPa}$ measured on plates at $4.2 \mathrm{~K}$. The MIG weldability of the alloy has been proven and specified. Moreover, the $R p_{0.2}$ of the joint at 4.2 $\mathrm{K}$, produced with an EN AW-5556 filler, has been measured between $210 \mathrm{MPa}$ and $233 \mathrm{MPa}$, which is fully satisfactory for the application [13].

Large plates 75-mm thick in EN AW-5083-H321 (to be reduced in thickness to $50 \mathrm{~mm}$ by machining after welding) are industrially available and certified according to ASTM B209M. Nevertheless this standard covers, for plates in H321 temper, a thickness up to only $80 \mathrm{~mm}$ guaranteeing a minimum Room Temperature (RT) $R p_{0.2}$ of $200 \mathrm{MPa}$. Since the strength decreases with increasing thickness, the properties associated to this temper would no longer be certified nor adequate for the flanges or the shoulders thicker than $80 \mathrm{~mm}$.

As an alternative to a welded construction, the ten end-flanges, and the shoulders that are not integrated into the thickness of the shells, are obtained from seamless rings in the same alloy. 
The selection of the ring rolling technique for thick components resulted in fully satisfactory properties [14], with improved level of homogeneity and isotropy of tensile properties compared to thick plates (minimum $R p_{0.2}$ measured at $4.2 \mathrm{~K}$ in the axial direction over $270 \mathrm{MPa}$ ), exceptional ductility in the longitudinal direction even increasing with decreasing $\mathrm{T}$ (from $18 \%$ at RT to $28 \%$ at $4.2 \mathrm{~K}$ ).

\section{Manufacture of Mandrels}

Almost all large solenoids constructed to date have used mandrels made up from bent plates in EN AW-5083-O (fully annealed). Several intermediate stress-relieving thermal heat-treatments were applied during manufacture to relax stresses and fulfill the requirements of geometrical tolerances. This is not possible anymore if one wants to maintain the mechanical properties of the EN AW-5083-H321up to the end.

Ansaldo-Superconductori has used a technique of construction in which referencing tooling is used, either inside or outside the mandrels, to allow machining, respectively, outside or inside. Then, keeping the outer tooling, winding can proceed. Once winding is completed, its stiffness is sufficient to maintain the cylindricity of the ensemble within the $10 \mathrm{~mm}$ range, and the outer referencing tooling can be removed.

A construction foreseeing seamless flanges and welded shells helps avoiding stress relieving, compared to a full welded construction, since the seamless rings represent also a good dimensional reference for each module.

\section{E. Winding a Stiff Conductor}

The inner winding of such a stiff conductor was a worry, but, in the end, after a dedicated pre-industrialization activity, it proved easy to execute using the ad-hoc winding machine designed and built by Ansaldo-Superconductori [15].

\section{F. Limiting the Shear Stress Inside the Coil}

Controlling the strain and keeping the shear stress low everywhere inside the cold mass has been a continuous driving goal of the design [16].

Regions for potential high shear stress lie between modules because the full compression force must be transmitted from module to module in a very discontinuous region. To alleviate this difficulty a 220-ton coil in one unit was even considered for some time, but this design was abandoned as too ambitious. In particular, it was not possible to obtain Rutherford cables longer than $3 \mathrm{~km}$ without junctions.

A novel design has been suggested by Ansaldo-Superconductori. It uses a set of wedges to terminate each layer so that the end of each module is already fairly flat. The subsequent gluing, after impregnation, of $5 \mathrm{~mm}$ thick G11 plates and the final machining of these plates, using a vertical lathe, allowed improving the flatness thus permitting a perfect coupling from module to module [17].

In fact, the use of a dedicated workshop to construct the mandrels nearby the winding area has proved to be a tremendous asset. Not only it saved numerous difficult transports of large pieces, but also it allowed the machining of the top flange region of each 50-t module after impregnation.

\section{G. Coil Assembly}

For final assembly, the coupling of the modules with their axes horizontal was abandoned because the shape of each module was difficult to control during this operation. Finally it was decided to stack the modules with their axes vertical ensuring a good coupling between modules, thus allowing reconstructing the full coil in the vertical position without any deformation.

Then, taking advantage of the very large stiffness of the coil, the complete coil has been rotated by 90 degrees, cantilevered from one end, to allow insertion inside the outer vacuum tank. The same large tooling was used to insert the inner vacuum tank inside the coil [2].

\section{H. Quality Control}

Having to reject any element far down in the manufacturing process has so dramatic an implication on such large projects that it must be avoided at all cost.

Structured quality control and traceability has been an important continuous action in particular for all conductor elements, the finished conductor, the construction of the mandrels, the insulation, the winding and the impregnation.

\section{Project Organization}

The construction of such a magnet is beyond the capabilities of any high-energy physics laboratory. The solution retained by CMS has been the creation of an ad-hoc "coil collaboration" allowing regrouping international laboratories, having complementary expertise in the necessary fields, and leading working groups for each main task of the project. The management structure has been adapted to reflect it, and all technical and procurement decisions have been proposed by the Project Manager and endorsed by the Magnet Technical Board (MTB) in which all participating laboratories were represented. This has allowed a very integrated project in the framework of a truly international collaboration.

\section{CRitical Review of Retained SOlutions AND THEIR RELEVANCE FOR DESIGNING A NEW 4 OR 5-T COIL}

Applying the same general design for a large thin coil generating DC field, for particle detector for example, seems possible. In particular the electrical performances of $\mathrm{NbTi}$ are such that a 5-T coil can be designed following the same principles, possibly with some variations.

\section{A. Stability Margin Considerations}

Designing for an enthalpy stability margin exceeding $1.5 \mathrm{~K}$ seems prudent to keep. This is one of the most difficult parameter to apprehend and keep under control as the actual margin depends of possible local defects during the construction or operation [18], like section of superconducting wire below specification, or bad bonding of the Rutherford cable, or cracking of the epoxy resin. In fact, during the testing of the CMS coil, being at $4 \mathrm{~T}$, the temperature of the coil has been allowed to drift by $0.7 \mathrm{~K}$, up to $5.5 \mathrm{~K}$, without inducing any quench [4], showing that the coil has a sufficient stability margin but it does not demonstrate that this margin can be safely reduced. Keeping 
a stability margin exceeding $1.5 \mathrm{~K}$ just means putting more superconducting wires and this is negligible as far as price is concerned.

The stability with respect to localized disturbances depends also of the electrical resistivity of the stabilizer under field and under stress. It is known that each stressing cycle decreases the RRR of the stabilizer [19]. In fact the stability margin computations for the CMS coil have been performed for a degraded pure aluminum with an apparent RRR of 400 under field. Thus the drive to procure a super pure aluminum with a RRR of 3000 at zero field and no stress seems a little vain.

\section{B. Compound Reinforced Conductor Considerations}

To use a compound conductor for large high field, indirectly cooled, potted solenoids seems still a good solution. It allows incorporating in an easy way the quantity of high-strength alloy required by strain considerations.

The first alternative to the compound reinforced conductor is to have a thick mandrel, but as discussed above, such a thick mandrel seems very difficult to manufacture by welding with the necessary mechanical properties. Furthermore, in such a solution, one loses the advantage of reacting the magnetic force where it is created leading to dangerous shear stress inside the coil, in particular at the winding/mandrel critical bonding region.

Another alternative to the compound conductor is to wind an aluminum alloy reinforcement section in parallel to the conductor proper. This alternative has been rejected by the design team, at the time, as it would render the winding process much more complicated, giving a winding of inferior quality with more fragile and numerous interfaces filled with fiberglass epoxy or pure resin and it is known that these are really the weak points of potted coils. The module-to-module coupling would also be also be much more difficult to make as one would have to deal with two distinct components. The only advantage of such and independent galvanic element would be its possible use as efficient quench detector.

\section{Is it Necessary to Improve the Mechanical Properties of the Alloys for the Reinforcement and the Mandrels?}

When planning to increase the induction of a large thin coil, an important question arises concerning the choice of the reinforcing alloy. Do more performing grades have to be used? The extrudable and weldable alloy EN AW-7020 could be a candidate, despite higher extrusion costs, if extrusion in long lengths would prove satisfactory. However, the alloys used for CMS for the mandrels or the reinforcement are in fact sufficiently performing for the next generation of coils, and thus it is not necessary to look for stronger alloys.

This is coming from the fact that the stored magnetic energy per unit of volume inside the thin solenoid in $\mathrm{J} / \mathrm{m}^{3}$, and the equivalent pressure acting inside the thin solenoid in $\mathrm{N} / \mathrm{m}^{2}$ can both be expressed by the same expression: $B^{2} /\left(2 \mu_{0}\right)$, in which $B$ is the central induction in $\mathrm{T}$ and $\mu_{0}$ is the permeability of vacuum. If $\alpha$ is the ratio of structural aluminum alloy inside the coil, $Y$ its Young's modulus, and $\rho$ the density of aluminum (pure and alloy), then, neglecting the mechanical effect of the pure aluminum and applying the formula to compute stress in pressurized thin tubes, one can relate the magnetic energy per unit mass of the cold mass $E / M$ to the hoop strain $\varepsilon$ of the coil by the relation:

$$
E / M=\alpha Y /(2 \rho) \varepsilon .
$$

Thus, the hoop strain is also a measure of the stored energy per unit mass of the cold mass.

It seems difficult, respecting construction codes, to exceed a hoop strain of $0.15 \%$. In the case of CMS, this corresponds to a maximum Von Mises stress of $140 \mathrm{MPa}$, requiring alloys with $R p_{0.2}>210 \mathrm{MPa}$ and $R_{\mathrm{m}}>420 \mathrm{MPa}$ at $4.2 \mathrm{~K}$.

In the other hand, one has to be careful to increase the ratio $E / M$ because, taking into account the enthalpy of aluminum, this is representative of the mean temperature of the coil after a fast dump. In the case of CMS, as 50\% of the energy is extracted and dissipated in the dump resistors, this corresponds to a safe mean temperature of $80 \mathrm{~K}$ for the cold mass. It covers also the ultimate accidental case, the one in which an active element of the dumping system is not working and all the energy is dissipated in the cold mass by the passive quench-back protection scheme.

Thus one can tentatively conclude that the selected alloys EN AW-6082-T51 for the reinforcement and EN AW-5083-H321 for the mandrels are perfectly suitable for a 5-T coil designed for ultimate reliability, even in case of failure of an active element of the protection system.

\section{Improving the Compound Reinforced Conductor by Replacing the Pure Aluminum Stabilizer}

The considerations on stability margin open the door to using a less performing stabilizer in terms of RRR in the virgin state, but more performing in terms of mechanical properties.

There is a development project [20] to manufacture $200 \mathrm{~m}$ of an improved CMS reinforced conductor in which the pure aluminum is replaced by the doped alloy $\mathrm{Al}-0.1 \mathrm{wt} \% \mathrm{Ni}$ developed by KEK for the ATLAS thin solenoid [21]. This alloy in the cold work state reaches a yield strength $R p_{0.2}$ of $85 \mathrm{MPa}$ at $4.2 \mathrm{~K}$, even after a $130^{\circ}-15 \mathrm{~h}$ curing cycle, while maintaining a RRR of 590 .

This new compound conductor would be more performing mechanically as in the preceding formula the factor $\alpha$ would be nearly 1 . Another clear advantage would be of staying in a nearly fully elastic state, thus exhibiting constant properties with time in terms of mechanical properties and RRR [22].

\section{E. Attaching the Reinforcement Sections to the Insert}

The way the reinforcement sections are attached to the insert can certainly be reviewed. The EB welding process may look like a deluxe one, although it has numerous advantages: the quality is superb and the process is easy to monitor and it can be stopped and re-started at any moment. In the end the EB welding process proved easy to apply.

To have in hand an alternative to EB welding the design team considered for a short time the possibility of attaching the reinforcement sections by continuous soft soldering. This looked promising but, by lack of available effort, this study was stopped as soon as the EB solution proved able to succeed. 


\section{F. Cooling Circuits and Joints}

The mandrels being strained at $0.15 \%$ are not stationary structures. This could induce stresses in the superconducting joints if they are attached to the mandrel in the traditional way to profit from the heat sink. This was recognized late in the project and the joints were rendered free. Adding pure aluminum braids connecting the joint holders to the mandrels ensured good cooling.

The same problem was identified for the thermosiphon circuits, which originally were connected too stiffly to the mandrels, risking overstressing the numerous pipe-to-pipe welds. In fact the thermosiphon system has been doubled for redundancy, as a leak discovered late is practically impossible to repair in a reasonable time. More than 600 welds have been performed to connect aluminum pipes and discovering a leak when at helium temperature was considered one of the main risks of the full project. This risk could be mitigated by using stainless manifolds and aluminum/stainless-steel couplings allowing performing only stainless-steel-to-stainless-steel butt welds on site.

\section{CONCLUSIONS}

At the start of the CMS coil project, in early 1990's, several problems were identified but had no known solution, and the construction proper even started with some of these problems still in the pending state. It took years to develop and validate some of the retained solutions.

Problems connected to superconductivity were quickly solved. In fact, looking backwards, it appears that most of the difficult problems were connected to mechanics, stress and strain distribution, and procuring components with the necessary mechanical properties, in the required size for mandrels, or along the full conductor lengths. With the drive to increase the field, at least for some of the future projects, these mechanical problems are likely to become even more dominant. New processes can be developed, however, the passing of the superconductor through all the manufacturing processes imposes severe constraints to these processes not to degrade the electrical performances of the superconductor.

Knowing what we know today, and asking ourselves what would we change if we had to build a new large high-field thin superconducting coil, we, as a group, concluded that we would basically use what is now referred to as the CMS design, with the possible following improvements: replace the pure aluminum stabilizer by a doped alloy and look for an alternative to EB welding if it proved to be clearly less expensive.

Apart for these points, the general design would be basically extended as is; one of the basic reasons to resist changes being the fact that one cannot risk constructing a non-performing large coil by introducing more risky solutions just because they are potentially less expensive.

Alloys selected for CMS, EN AW-6082-T51 for the reinforcement and EN AW-5083-H321 for the mandrels are perfectly suitable for a 5-T coil designed for ultimate reliability even in case of failure of one active element of the protection system.

The CMS design, which is clearly adapted to DC fields staying within the range of NbTi with a sufficient enthalpy stability margin at $4.5 \mathrm{~K}$, can thus be extrapolated up to $5 \mathrm{~T}$.

More demanding solenoids, designed for higher fields for example, would necessitate a different approach, like the use of cable-in-conduit technology profiting from future technical developments coming from the ITER project.

\section{REFERENCES}

[1] CMS Magnet Collaboration, The Magnet Project: Technical Design Report CERN, CMS TDR 1, 1997, vol. CERN/LHCC97-10.

[2] B. Levesy et al., "CMS solenoid assembly," IEEE Trans. App. Superconduct., vol. 16, pp. 517-520, Jun. 2006.

[3] D. Campi et al., "Commissioning of the CMS magnet," IEEE Trans. App. Superconduct., vol. 17, no. 2, June 2007.

[4] F. Kircher et al., "Magnetic tests of the CMS superconducting magnet," in Proc. 20th Int. Conf on Magnet Technology, Philadelphia, 2007, submitted for publication.

[5] V. Klyukhin et al., "Measurement of the CMS magnetic field," in Proc. 20th Int. Conf on Magnet Technology, Philadelphia, 2007, submitted for publication.

[6] J. M. Baze et al., "Design construction and test of the large superconducting solenoid ALEPH," IEEE Trans. Magn., vol. 24, pp. 1260-1263, 1988.

[7] J. C. Lottin et al., "Conceptual design of the 4 T CMS solenoid," in Proc. of Int. Cryogenic Material Conf., New York, 1996, vol. 41 A, pp. 819-826, Advance in Cryogenics Engineering, Plenum Press.

[8] I. L. Horvath, "Superconducting cable development for future high energy physics detector magnets," in Proc. of 4th Int. Conf. on Advanced Technology, 1995, vol. 44, pp. 672-676, Nucl. Phys. B.

[9] I. L. Horvath, "Development of an aluminum stabilized reinforced superconducting conductor," IEEE Trans. Magn., vol. 32, pp. 2200-2202, 1995.

[10] R. Folch et al., "The CMS conductor," IEEE Trans. App. Superconduct., vol. 12, pp. 345-348, 2001.

[11] S. S. Tavares et al., "An improved billet on billet extrusion process of continuous aluminum alloy shapes for cryogenic applications in the Compact Muon Solenoid experiment," Journal of Materials Processing Technologies, vol. 143-144, pp. 584-590, Dec. 2003.

[12] B. Curé et al., "Mechanical properties of the CMS conductor," IEEE Trans. App. Superconduct., vol. 14, pp. 530-533, 2004.

[13] M. Castoldi et al., "Possible fabrication techniques and welding specifications for the external cylinder of the CMS coil," IEEE Trans. App. Superconduct., vol. 10, pp. 415-418, Mar. 2000.

[14] S. Sgobba et al., "Design, construction and quality tests of the large Al-alloy mandrels for the CMS Reinforced Conductor," IEEE Trans. App. Superconduct., vol. 12, pp. 428-431, Mar. 2002.

[15] P. Fabbricatore et al., "The winding line for the CMS reinforced conductor," IEEE Trans. App. Superconduct., vol. 12, pp. 358-361, Mar. 2002.

[16] A. Desirelli et al., "Finite element stress analysis of the CMS magnet coil," IEEE Trans. App. Superconduct., vol. 10, pp. 419-423, Mar. 2000.

[17] P. Fabbricatore et al., "The manufacture of modules for CMS coil," IEEE Trans. App. Superconduct., vol. 16, pp. 512-516, Jun. 2006.

[18] P. Fabbricatore et al., "Experimentl study of CMS conductor stability," IEEE Trans. App. Superconduct., vol. 10, pp. 424-427, Mar. 2000.

[19] B. Seeber et al., "Variation of the residual resistivity ratio of the aluminum stabilizer for the CMS conductor under dynamic stress at 4.2 K," IEEE Trans. App. Superconduct., vol. 10, pp. 403-406, Mar. 2000.

[20] P. Campi, Jul. 2007, (private communication).

[21] K. Wada et al., "Development of high-strength and high-RRR aluminum-stabilized superconductor for the Atlas thin solenoid," IEEE Trans. App. Superconduct., vol. 10, pp. 373-376, Mar. 2000.

[22] S. Sgobba et al., "Toward an improved high strength, high RRR CMS conductor," IEEE Trans. App. Superconduct., vol. 16, pp. 521-524, Jun. 2006. 